\title{
How the Sleep of Couples Changes from Pregnancy to Three Months Postpartum
}

This article was published in the following Dove Press journal:

Nature and Science of Sleep

\section{Barbara G Cattarius $\mathbb{D}$ Angelika A Schlarb}

Faculty Psychology and Sports Science, University of Bielefeld, Bielefeld, Germany
Correspondence: Barbara G Cattarius University of Bielefeld, Faculty of Psychology and Sports Science, P.O.P, I0013I, Bielefeld, 33015, Germany Email B.Cattarius@uni-bielefeld.de
Background: Sleep disturbances are frequent during pregnancy and postpartum. However, detailed research of sleep in couples during pregnancy and postpartum is lacking.

Objective: Changes of sleep for primi- and multiparous pregnant women and their partners from late pregnancy to three months postpartum. The particular focus of this study is on sex differences in sleep, sleep problems, mutual sleep influence of couples, and the influences of parity and feeding methods on couples' sleep.

Materials and Methods: The sample included 69 pregnant couples in the last trimester of pregnancy (t1) and three months after birth (t2). Sleep was measured with sleep diary for both times of measurement. The Pittsburgh Sleep Quality Index (PSQI) assessed sleep disturbances. Other variables as parity and infant feeding type were determined by questionnaire. Besides results for woman and men, also dyadic data are calculated.

Results: Over the time women had a worse sleep quality than men. They had a prolonged sleep onset latency, higher frequency and longer duration of night wakings than men. Sleep efficiency for women was prepartal $83.32 \%$ and postpartal $83.6 \%$ below the clinically cut-off value of $85 \%$. For $56.52 \%$ of women at $\mathrm{t} 1$ and for $55.07 \%$ at $\mathrm{t} 2$ PSQI scores exceeded the clinically cut-off of 5 . However, men suffered from a sleep loss after birth of their child, too. In pregnancy and postpartum men reported lower total sleep time at both times of measurement in comparison to women. For $30.43 \%$ of men at T1 and for $24.64 \%$ at T2 PSQI score exceeded the clinically cut-off of 5. Sleep efficiency for men was prepartal $90.96 \%$ and postpartal $90.69 \%$. Results indicate predictive links between prepartal PSQI of couples to postpartal PSQI. Neither parity nor feeding method could explain variance in postpartal PSQI-score.

Conclusion: This is one of the very rare studies incorporating dyadic data. Results show the need of diagnosing and treating existing sleep problems in pregnancy to prevent future sleep problems postpartum.

Keywords: sleep, couples, pregnancy, postpartum

\section{Introduction}

Research on sleep in women and men has been ongoing for many decades, and gender differences in sleep regulation are well known. Non-pregnant women often have higher total sleep time at night (TST), more slow-wave sleep, less Phase 1 sleep, and longer REM latency than men. ${ }^{1}$ Various authors suggested that this is due to the beneficial effects of gonadal hormones on women. ${ }^{1}$ These typical gender differences in sleep architecture often begin with puberty at ages 14-17 years. ${ }^{18,28}$ A large panel study with questions about sleep behavior also demonstrated that women reported lower sleep satisfaction than men, although their sleep duration 
was comparable. ${ }^{23}$ Therefore, gender must be considered a major risk for insomnia or sleep complaints..$^{15,18}$

The transition to parenthood can be a stressful life event for many couples, with far-reaching changes in sleep patterns. Thus, sleep is the focus of many research efforts, particularly during pregnancy and postpartum. Researchers emphasize the need for early detection of sleep disorders to prevent chronification of sleep problems. $^{24}$

\section{Prepartal and Postnatal Sleep of Women}

The sleep of pregnant women in the last trimester of pregnancy is often disturbed compared to non-pregnant women. Reported were significant differences in sleep efficiency (77.6\% vs $91 \%)$ and increased sleep fragmentation in the form of frequent awakenings. ${ }^{8}$ Other authors found difficulties falling asleep and staying asleep and frequent awakenings at night, ${ }^{7,17}$ TST also changes over the course of pregnancy and after delivery. While TST was $7.8 \mathrm{~h}$ in the last trimester, it was shortest postpartum at $7 \mathrm{~h}$, and $20.5 \%$ of the women rated their sleep as restless. ${ }^{7}$ Nevertheless, for many women, sleep improves postpartum with a decrease in sleep onset latency (SOL) (from 8.4 $\min$ to $4.2 \mathrm{~min}$ ) and frequency of night waking (FNW) and an increase in sleep efficiency (SE) $(75.2 \%$ to $96 \%)$ as demonstrated by other authors. ${ }^{8}$

Postnatal factors, such as first vs second child or breastfeeding vs bottle feeding, may cause differences in postpartal sleep. Primiparae were reported to suffer from a significant reduction in SE compared with multiparae $(77.25 \%$ vs $87.7 \%){ }^{31}$ The authors attributed this to firsttime parents (primiparous couples) being less experienced in managing a newborn compared with experienced parents (multiparous couples) and therefore they were more likely to suffer from sleep problems. ${ }^{31}$ One study contradicted these findings with a higher FNW and lower SE for multiparous parents. ${ }^{12}$ The majority of studies associate breastfeeding with better sleep quality for mothers, primarily related to the fact that breastfeeding mothers frequently co-sleep and return to sleep more quickly. One study, for example, emphasizes the many benefits of breastfeeding for both mother and baby during cosleeping, with increased TST for breastfeeding mothers $\left(5.9\right.$ h). ${ }^{21}$ In contrast, total sleep time during bottlefeeding $(5.7 \mathrm{~h})$ was unaffected by the baby's sleep location. $^{21}$

While numerous studies have investigated many factors influencing prenatal and postnatal sleep in women, only a very limited number of studies have investigated and integrated sleep in expectant fathers and fathers both during pregnancy and postpartum. ${ }^{4,5,20,23,31}$

\section{Prepartal and Postpartal Sleep of Couples}

Elek and colleagues have shown that women's sleep is more impaired than men's in both pregnancy and postpartum. In the last trimester of pregnancy, men had a higher TST than women $(15.8 \mathrm{~min}){ }^{4}$ Another study examined couples both prenatally and postnatally and showed a decrease in TST at night from prenatal to postpartum in women, while TST increased in men (women: -40min./ men: $+13 \mathrm{~min}) .{ }^{31}$ In addition, women interacted with the infant at night more often than men, with consequently less rest at night in mothers. ${ }^{32}$ Other authors found a decrease in TST from prepartum to postpartum for both sexes (women/men: $-41.2 \mathrm{~min} /-15.8 \mathrm{~min})^{5}$ WASO increased in both parents, but mothers were more affected than fathers, both in pregnancy and at postpartum. ${ }^{5}$ Another study assessed data three months after birth and found an average of 40-45 min more total sleep time at night in first-time parents of exclusively breastfed newborns compared to bottle-fed newborns. ${ }^{3}$ A large panel study with questions on sleep behavior also showed that women reported lower sleep satisfaction than men, although their sleep duration was comparable. ${ }^{23}$ Postnatally, both sexes reported lower sleep satisfaction with shorter sleep periods compared to the prenatal survey period. ${ }^{23}$

In addition, other studies on couples can be found, but the data in these studies were collected postpartum only. For example, one study found higher FNW and poorer sleep quality in mothers compared to fathers, which was decisively predicted by the frequency of the child's nighttime awakenings. ${ }^{14}$ Others reported lower sleep quality in first-time mothers compared with first-time fathers, with higher FNW and longer periods awake (WASO) at night in mothers (FNW mothers/fathers: 3.3/2.4; WASO mothers/ fathers: $116 \mathrm{~min} / 42.7 \mathrm{~min}){ }^{10}$

Although sleep disturbances were more frequently observed in mothers in the aforementioned studies, men are also negatively affected in their sleep behavior by the nocturnal needs of the newborn, and $10 \%$ of first-time fathers reported sleep deprivation as a major stressor. ${ }^{20}$ Therefore, sleep and any sleep problems in women and men should be considered from a dyadic perspective, especially when a couple shares a bedroom both prenatally and postnatally. 


\section{Purpose}

The present study is intended to fill a research gap concerning sleep of couples in the transition to parenthood, since only very few studies have also taken fathers into account. Although sleep studies of couples can be found, only a rare number of studies included pre- and postnatal measurement time points or comparable evaluation methods and did not implement dyadic data discriminating differences between couples. Based on the literature, we hypothesize 1) that symptoms of disturbed sleep are more common in women than in their partner during pregnancy and postpartum as measured by the PSQI and a sleep diary, 2) that female and male sleep are strongly associated when they share a bedroom 3) differences between the couples-related data are explained by parity, and 4) differences between couples are explained by feeding method. In addition, we hypothesize that 5) there is an association between pre- and postpartum sleep quality in couples, demonstrated by membership in a pre- and postpartum sleep group.

\section{Methods}

\section{Procedure}

A natural sample of 87 pregnant couples was recruited in Germany via advertising at prenatal classes, in newspapers, and with information sheets. A research assistant visited the participants in the last trimester of pregnancy to explain goals and procedure of the study at hand. All couples were instructed about questionnaires and sleep diary for ten nights. All participants also got an information letter explaining the background and purpose of the study and were informed that the data would be collected on an anonymous basis. Participation was voluntary; participants could withdraw from the study at any time without negative consequences. All participants signed informed consent before participation. The study was approved by the Ethics Committee of Bielefeld University and was in accordance with the Declaration of Helsinki.

\section{Participants}

A total of 174 participants - 87 couples (women and men) completed the questionnaires and a sleep report during the last trimester of pregnancy ( $\mathrm{t} 1$ ) (gestational age $=$ on average 35 weeks and 2.58 days) and three months postpartum ( $\mathrm{t} 2$ ) (see Figure 1). Although same-sex couples could participate in the study, only heterosexual couples participated. Eighteen couples had to be excluded from the study as they did not complete the questionnaires at $\mathrm{t} 2$. The final sample consisted of 69 couples. The sample was homogeneous in terms of race (100\% white individuals). Of the participating couples, $45(65.22 \%)$ were having their first child ( 45 women: age range $=22-40$ years, $M=30.38, S D=4.63 ; 45$ men: age range $=26-52$ years, $M=33.30, S D=5.42)$ and 24 couples $(34.78 \%)$ were having their second or third child ( 24 women: age range $=26-39$ years, $M=33.54, S D=3.62 ; 24$ men: age range $=26-45$ years, $M=36.58, S D=4.59$ ).

In terms of educational level, $48.9 \%$ held an A-level certificate, $23.6 \%$ held a university of applied science degree, whereas $15.7 \%$ held a highschool certificate and $8.4 \%$ had completed junior highschool. Two participants had no educational degree. Nobody reported a critical illness. Concerning birth procedures, 42 women had a vaginal delivery, 23 women had a caesarean section, and four women had a vaginal operative birth. Fifty-four babies (78.3\%) were breast-fed and 15 babies (21.7\%) were bottle-fed and drunk formula. All babies slept in their parents' bedrooms.

\section{Measures of Sleep Pittsburgh Sleep Quality Index (PSQI)}

The women and men completed the German version of the Pittsburgh Sleep Quality Index (PSQI) during pregnancy and postpartum. The PSQI is a widely used and wellvalidated 19-item self-report instrument measuring sleep disturbances in adults. ${ }^{2}$ It includes seven subscale scores (sleep quality, sleep latency, sleep duration at night, habitual sleep efficiency, sleep disturbances, sleep medications, and daytime dysfunction) and provides a global score, ranging from no sleep difficulty to severe difficulties. Scores range from 0 to 21. A global score $>5$ indicates "poor sleep"; it has been shown to have a diagnostic sensitivity between $80 \%$ and $90 \%$ and specificity between $83 \%$ and $87 \%$.

\section{Sleep Diary}

All participants had to fill in a sleep diary of the German Sleep Society (DGSM), ${ }^{9}$ at both time of measurement: first, in pregnancy and second, in postpartum. The sleep diary was used to investigate sleep-related characteristics over a ten-day period, such as time of going to bed, SOL, FNW, WASO, nocturnal feeding (postpartum), TST. Based on this information, SE (ratio of minutes of sleep divided by number of minutes in bed) and overall TST were calculated. A statistical evaluation of this instrument revealed that it discriminates well between normal sleepers 


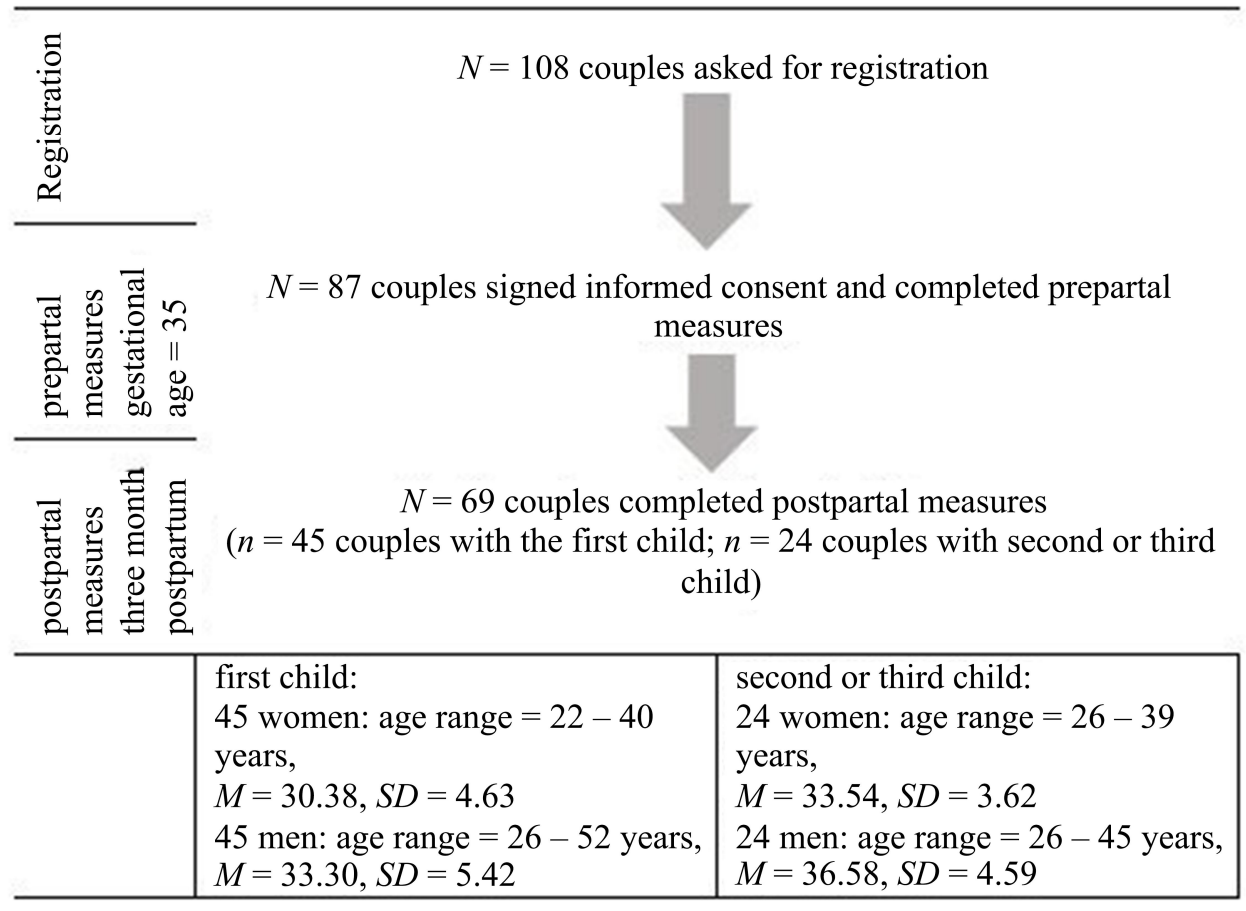

Figure I Number of participants, dropout and age distribution of the participants.

and patients suffering from insomnia. Its reliability and validity were also acceptable. Sleep diaries are widely used and reliable and valid. ${ }^{9}$ In addition, they adequately correlate with objective sleep measures, such as polysomnography (kappa $=0.87$ ), and show high sensitivity $(92.3 \%)$ and specificity $(95.6 \%) .^{25}$

4During each measurement point of the study, women and men completed the PSQI and a sleep diary. All couples completed the sleep diary for ten days at both times of measurement. The study began on average at 35 weeks gestation ( \pm 2.58 days) for ten consecutive days (t1). The second measurement began 12 weeks postpartum for ten consecutive days ( $\mathrm{t} 2)$.

\section{Statistical Analysis}

SPSS version 25 (SPSS, Inc., Chicago, IL) for Windows was used to calculate descriptive statistics. For comparison between women and men, paired $t$-tests were conducted at both times of measurement. Chord diagram and modelling were performed using $\mathrm{R}$ version 4.0.1. ${ }^{22} P$-values were reported using a $5 \%$ significance level. Due to the explorative nature of the study no adjustment of $p$-value for multiplicity was performed. The influence of prepartum sleep parameters on postpartum sleep parameters (PSQI, SOL, FNW, WASO, TST and SE) were analyzed using the Actor-
Partner Interdependence Model (APIM) with distinguishable dyads allowing for modeling the influence that members of a couple have on each other. ${ }^{11}$ Parameters of the APIM were estimated using structural equation modeling. ${ }^{26,30}$ In addition, the relative sizes of the actor and partner effects are analyzed by calculation of the parameter $\mathrm{k}$ as ratio of the partner to the actor effect and $95 \%$ confidence intervals of $\mathrm{k}$ are determined by means of Monte Carlo sampling. Furthermore, parity and feeding methods were included as covariates in the model for the PSQI.

For the given research question the APIM is visualized in the following diagram, see Figure 2.

Actor effects exists when the association is within person, eg mother's prepartum sleep parameter will predict mother's postpartum sleep parameter. Partner effect exists when the association is between the couple (eg father's prepartum sleep parameter will predict mother's postpartum sleep parameter). The relative sizes of the actor and partner effect and 95\% bootstrapped confidence intervals (CI) will be calculated and will be used for determination which model (ie actor-only, couple or contrast) fits best.

For further analyses, the PSQI were relevant. To compare quality of sleep, a global PSQI score $\leq 5$ indicated a good sleeper and a PSQI score $>5$ a poor sleeper. For the dyadic data, each couple was assigned to a sleep quality group in pregnancy. If both 


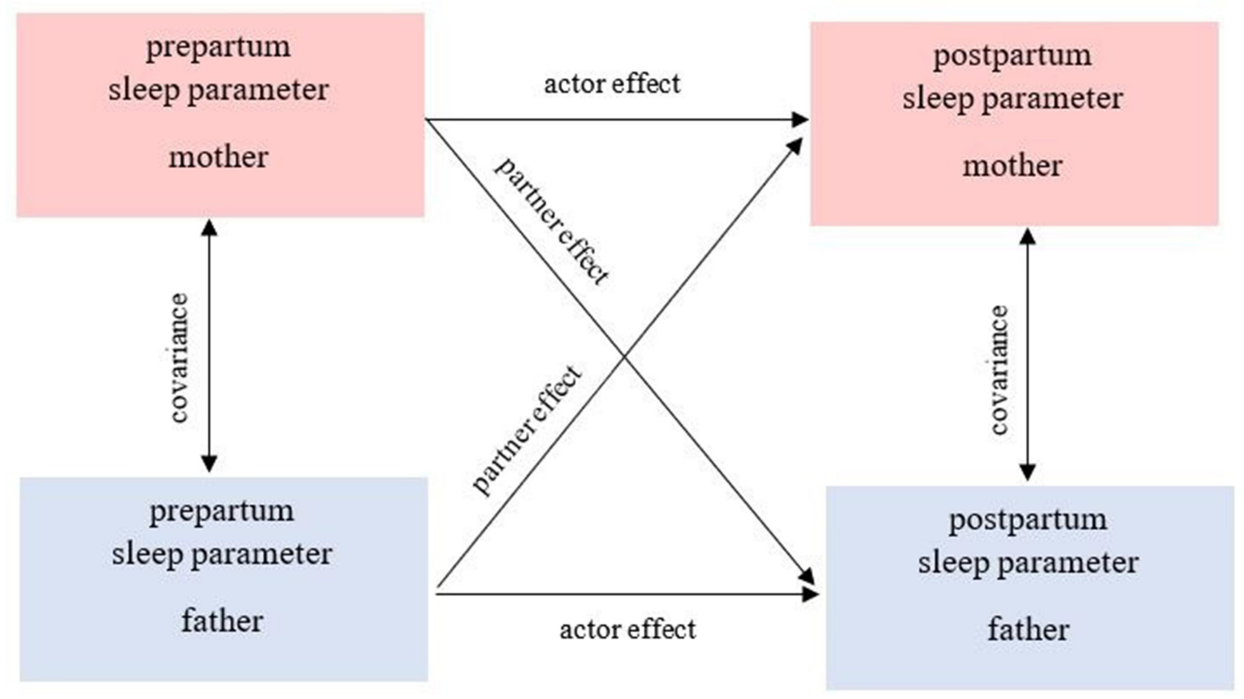

Figure 2 Actor-partner-interdependence-model (APIM).

parents were good sleepers based on the aforementioned PSQI cut-off scores, they were assigned to group 1, which will henceforth be called "both good sleepers". If one of the parents was a "poor sleeper", the couple was assigned to group 2: the "one person is a bad sleeper" group. If both parents were "poor sleepers", they were assigned to group 3: "both bad sleepers".

The transitions from prepartum to postpartum sleeping groups are visualized using a Chord diagram, ${ }^{6}$ which represents flows or connections between several entities. Each entity is represented by a fragment on the outer part of the circular layout. Then, arcs are drawn between each entity. The size of the arc is proportional to the importance of the flow.

\section{Results}

Table 1 displays descriptive data and results concerning differences in women and men at both times of measurement for PSQI, SOL, TST at night, WASO, FNW and SE.

Women were more sleep impaired compared to men at both time of measurement. Both prepartum and postpartum, women had a significantly higher PSQI above the clinically significant cut-off value of 5 compared to men, placing women among the poor sleepers both prepartum and postpartum. In addition, women had a significantly higher SOL and a significantly higher FNW than men. SE in women was significantly decreased compared with men at both measurement time points and was below the clinically relevant cutoff value of $85 \%$ in the range of insomniac disorder. In contrast, TST was significantly lower for men compared with women at both times of measurement. In addition, from prepartum to postpartum, men's PSQI increases slightly and men were consequently more impaired in their sleep postpartum than prenatally.

In the next step, APIM Analyses were performed for all sleep parameters.

\section{Results from APIM Analysis}

Table 2 displays APIM Analysis for all prepartum sleep parameters on postpartum sleep parameters. For detailed information a supplementary table (Supplementary Table$\mathrm{S} 1$ and supplementary figures (Supplementary Figure-S1, Supplementary Figure-S2, Supplementary Figure-S3, Supplementary Figure-S4, Supplementary Figure-S5, Supplementary Figure-S6) are provided.

\section{PSQI}

Considering relative sizes of the actor and partner effects, it can be concluded, that for mothers the couple and the actor-only models are plausible $(k=0.85,95 \%$ CI $[-0.14-$ 1.84]) whereas for fathers the actor-only model is plausible $(k=0.17,95 \%$ CI $[-0.1-0.44])$. There is no significant difference between the relative sizes of mothers and fathers $(p=0.211)$.

\section{SOL}

Relative sizes of the actor and partner effects are estimated for mothers $(k=0.61,95 \%$ CI $[-1.44-2.66])$ and fathers $(k=1.11$, $95 \%$ CI $[-1.84-4.06])$. For both the confidence interval is very wide and therefore the most likely model cannot be determined. Mothers and fathers differed not significantly $(p=0.800)$. 
Table I Descriptive Data and Results Concerning Differences in Women and Men at Both Times of Measurement

\begin{tabular}{|c|c|c|c|c|c|c|c|c|c|}
\hline & & \multicolumn{2}{|l|}{ Women } & \multicolumn{2}{|l|}{ Men } & \multirow[b]{2}{*}{$t$} & \multirow[b]{2}{*}{$d f$} & \multirow[b]{2}{*}{ Sig. } & \multirow[b]{2}{*}{ Cohen's o } \\
\hline & & $M(S D)$ & Range & $M(S D)$ & Range & & & & \\
\hline \multirow[t]{2}{*}{ PSQI score } & Pre & $6.67(2.88)$ & $2-14$ & 4.71 (2.09) & $0-10$ & $4.72^{* * * *}$ & 68 & 0.000 & 0.57 \\
\hline & Post & $6.03(2.63)$ & $1-14$ & $5.19(2.83)$ & $1-13$ & $2.20 *$ & 68 & 0.031 & 0.26 \\
\hline \multirow[t]{2}{*}{ SOL minutes } & Pre & $20.91(12.60)$ & $3-57$ & $13.38(8.77)$ & $\mathrm{I}-36$ & $3.81^{* * *}$ & 66 & 0.000 & 0.47 \\
\hline & Post & $14.09(9.53)$ & $\mathrm{I}-53$ & $11.55(1.00)$ & $\mathrm{I}-45$ & 1.88 & 68 & 0.064 & 0.23 \\
\hline \multirow[t]{2}{*}{ TST at night minutes } & Pre & $44 I .07(5 \mid .63)$ & $288-545$ & 418.81 (40.49) & $290-534$ & $3.34 * *$ & 67 & 0.001 & 0.41 \\
\hline & Post & $453.25(57.62)$ & $315-626$ & $432.52(7.26)$ & $27 \mid-597$ & $2.69 * *$ & 68 & 0.009 & 0.32 \\
\hline \multirow[t]{2}{*}{ WASO minutes } & Pre & $33.25(21.84)$ & $2-109$ & $10.45(13.17)$ & $\mathrm{I}-80$ & $7.36 * * *$ & 66 & 0.000 & 0.90 \\
\hline & Post & $39.25(24.54)$ & $1-103$ & II.07 (II.86) & $0-73$ & $10.02 * * *$ & 68 & 0.000 & 1.21 \\
\hline \multirow[t]{2}{*}{ FNW number } & Pre & $2.28(0.86)$ & $0.5-4.5$ & $0.95(0.08)$ & $0-2.6$ & $11.86 * * *$ & 67 & 0.000 & 1.43 \\
\hline & Post & $2.03(0.95)$ & $0.3-5.4$ & $1.02(0.08)$ & $0-2.9$ & $9.56^{* * * *}$ & 68 & 0.000 & 1.15 \\
\hline \multirow[t]{2}{*}{ SE percent } & Pre & $83.32(6.60)$ & $64.97-95.08$ & $90.96(4.78)$ & $68.32-98.34$ & $-7.5 \mathrm{I} * * *$ & 67 & 0.000 & 0.91 \\
\hline & Post & $83.60(6.60)$ & $62.47-96.17$ & $90.69(5.91)$ & $58.93-98.87$ & $-7.68 * * *$ & 68 & 0.000 & 0.93 \\
\hline
\end{tabular}

Notes: ${ }^{*} \mathrm{p}<0.05 . * * \mathrm{p}<0.01$. $* * * \mathrm{p}<0.0001$ (two tailed).

Abbreviations: PSQI, Pittsburgh Sleep Quality Index; SOL, sleep onset latency; TST, total sleep time; WASO, wake after sleep onset; FNW, frequency of night waking; SE, Sleep efficiency; pre, prepartal; post, postpartal.

Table 2 APIM Analysis for All Sleep Parameters

\begin{tabular}{|c|c|c|c|c|c|}
\hline & & \multicolumn{2}{|l|}{ Actor Effect } & \multicolumn{2}{|l|}{ Partner Effect } \\
\hline & & Mother & Father & Father $\rightarrow$ Mother & Mother $\rightarrow$ Father \\
\hline PSQI & $\begin{array}{l}\text { Estimate (std. Error) } \\
p \text {-value }\end{array}$ & $\begin{array}{l}0.331(0.099) \\
<0.001\end{array}$ & $\begin{array}{l}0.745(0.138) \\
<0.001\end{array}$ & $\begin{array}{l}0.282(0.139) \\
0.042\end{array}$ & $\begin{array}{l}0.128(0.098) \\
0.192\end{array}$ \\
\hline SOL & $\begin{array}{l}\text { Estimate (std. Error) } \\
p \text {-value }\end{array}$ & $\begin{array}{l}0.132(0.092) \\
0.150\end{array}$ & $\begin{array}{l}0.091(0.109) \\
0.408\end{array}$ & $\begin{array}{l}0.080(0.0132) \\
0.543\end{array}$ & $\begin{array}{l}0.101(0.075) \\
0.182\end{array}$ \\
\hline TST & $\begin{array}{l}\text { Estimate (std. Error) } \\
\text { p-value }\end{array}$ & $\begin{array}{l}0.591(0.115) \\
<0.001\end{array}$ & $\begin{array}{l}I .057(0.131) \\
<0.00 I\end{array}$ & $\begin{array}{l}0.166(0.149) \\
0.267\end{array}$ & $\begin{array}{l}0.113(0.101) \\
0.263\end{array}$ \\
\hline WASO & $\begin{array}{l}\text { Estimate (std. Error) } \\
p \text {-value }\end{array}$ & $\begin{array}{l}0.166(0.135) \\
0.220\end{array}$ & $\begin{array}{l}0.264(0.106) \\
0.013\end{array}$ & $\begin{array}{l}-0.132(0.222) \\
0.553\end{array}$ & $\begin{array}{l}-0.006(0.065) \\
0.932\end{array}$ \\
\hline FNW & $\begin{array}{l}\text { Estimate (std. Error) } \\
\text { p-value }\end{array}$ & $\begin{array}{l}0.410(0.128) \\
<0.001\end{array}$ & $\begin{array}{l}0.470(0.128) \\
<0.001\end{array}$ & $\begin{array}{l}0.234(0.172) \\
0.175\end{array}$ & $\begin{array}{l}0.038(0.095) \\
0.687\end{array}$ \\
\hline Sleep efficiency & $\begin{array}{l}\text { Estimate (std. Error) } \\
p \text {-value }\end{array}$ & $\begin{array}{l}0.460(0.107) \\
<0.001\end{array}$ & $\begin{array}{l}0.904(0.103) \\
<0.001\end{array}$ & $\begin{array}{l}0.127(0.147) \\
0.386\end{array}$ & $\begin{array}{l}0.068(0.076) \\
0.37 \mid\end{array}$ \\
\hline
\end{tabular}

Note: Bold values denote statistical significance at the $\mathrm{p}<0.05$ level.

Abbreviations: PSQI, Pittsburgh Sleep Quality Index; SOL, sleep onset latency; TST, total sleep time; WASO, wake after sleep onset; FNW, frequency of night waking.

TST

Considering relative sizes of the actor and partner effects, it can be assumed, that for mothers $(k=0.28,95 \% \mathrm{CI}$ $[-0.26-0.82])$ and fathers $(k=0.11,95 \%$ CI $[-0.09-0.30])$ the actor-only model is plausible. There is no significant difference between the relative sizes of mothers and fathers $(p=0.576)$.

\section{WASO}

Relative sizes of the actor and partner effects are estimated for mothers $(k=-0.79,95 \% \mathrm{CI}[-3.74-2.15])$ and fathers $(k=-0.02,95 \%$ CI $[-0.50-0.46])$. For mothers, the confidence interval is very wide and therefore the most likely model cannot be determined whereas for fathers the actor-only model is the most plausible 
model. No significant difference between the relative sizes of mothers and fathers is found $(p=0.600)$.

\section{FNW}

Considering relative sizes of the actor and partner effects, for mothers the couple and the actor-only models are plausible $(k=0.57,95 \%$ CI $[-0.41-1.55])$ whereas for fathers the actor-only model is plausible $(k=0.08,95 \%$ CI $[-0.33-$ $0.49])$. There is no significant difference between the relative sizes of mothers and fathers $(p=0.412)$.

\section{SE}

Considering relative sizes of the actor and partner effects, it can be concluded, that for mothers $(k=0.28,95 \% \mathrm{CI}[-0.35-$ $0.91])$ and fathers $(k=0.07,95 \% \mathrm{CI}[-0.09-0.24])$ the actoronly model is plausible. No significant difference between the relative sizes of mothers and fathers is found $(p=0.551)$.

\section{Results from APIM Analysis with Parity and Nutrition as Covariates}

In addition, a separate model with parity and type of child nutrition as covariates was conducted, see Figure 3.

Considering relative sizes of the actor and partner effects, it can be concluded, that for mothers the couple and the actoronly models are plausible $(k=0.84,95 \%$ CI $[-0.14-1.81])$ whereas for fathers the actor-only model is plausible $(k=0.16$, 95\% CI [-0.11-0.43]). No significant difference between the relative sizes of mothers and fathers is found $(p=0.208)$.

\section{Results from Chord Diagram}

To test if couples remain in the same sleep group after birth of their child (see Table 3), a chord diagram was created (see Figure 4).

As Figure 4 shows, of the 23 couples in sleep group 1 in pregnancy, $65.22 \%$ of couples $(n=15)$ remained in the same group three months postpartum. Similar observations were made in sleep group $2(50 \%=15$ couples $)$ and group $3(56.25 \%=9$ couples $)$. Furthermore, only 15 couples (21.74\% of the study group) moved to a better sleep group (group 1 or 2) and 15 couples $(21.74 \%$ of the study group) moved to a worse sleep group (group 2 or 3 ).

\section{Discussion}

\section{Comparison of Pre- and Postpartum Sleep in Women and Men}

The present study has succeeded in making a decisive contribution to revealing the sleep behavior of couples in the transition to parenthood. Thus, the stated aim of this study was to investigate the differences in sleep between women and men and also dyadic data both pre- and postpartum, taking into account various factors. Both prenatally and postpartum, women showed higher impairments in their sleep behavior than men, as measured by a higher PSQI score, a longer SOL, a higher FNW, and a longer WASO. With a PSQI that exceeded 5 at both measurement time points, $56.52 \%$ of women were above the clinically significant cut-off value of $5^{2}$ prenatally and $55.07 \%$ postnatally, and thus must be categorized as chronic poor sleepers. Whereas less men were above the cut-off value of the PSQI ( $30.43 \%$ prenatally and $24.64 \%$ postnatally). In addition, women's SE was below $85 \%$ at both time points and thus was below the clinically significant cutoff value, which is why their sleep must be considered as chronic disturbed. ${ }^{19}$ In contrast, men's sleep efficiency was in the normal range both before and after birth. The low SE in women was also reported in two other studies, ${ }^{16,31}$ although another study documented a postnatal increase of SE in women $(96 \%) .{ }^{8}$ In the present study, it was also important to highlight the change of sleep from prepartum to postpartum in both sexes. Both prenatally and postnatally, women had a higher TST compared with men. This result is consistent with findings of sex differences in sleep between women and men. ${ }^{1}$

In women, TST increased from $7.35 \mathrm{~h}$ during pregnancy to $7.55 \mathrm{~h}$ after delivery. Men also experienced a postpartum increase in TST from $6.98 \mathrm{~h}$ to $7.21 \mathrm{~h}$. In contrast to these results, one study found a decrease in TST in women from $7.8 \mathrm{~h}$ in the third trimester of pregnancy to $7.00 \mathrm{~h}$ in the first month postpartum. ${ }^{7}$ Beyond, while FNW decreased slightly for women in the present study, it increased slightly for men. This result is also in agreement with previous study findings who reported a decrease in FNW from pregnancy to postpartum in women. ${ }^{16}$ Furthermore, in our study, only $1.4 \%$ of pregnant women in the last trimester and $5.7 \%$ three months postpartum did not report nocturnal awakening, this is in line with one study (T1/T2: $1.9 \% / 4.7 \%){ }^{7}$ In addition, women's WASO increased from prepartum to postpartum (33.25 $\mathrm{min}$ to $39.25 \mathrm{~min}$ ), which was also found in previous studies, ${ }^{16}$ (31.5 $\mathrm{min}$ in pregnancy and $47.1 \mathrm{~min}$ postpartum). About $56.52 \%$ of women in the present study reported poor sleep or impaired sleep quality which is consistent with previous research, ${ }^{3,29}$ in which $45.2 \%$ to $53.5 \%$ of women suffered from decreased sleep quality. Nevertheless, it must be kept in mind that comparisons are limited because previous studies used different 


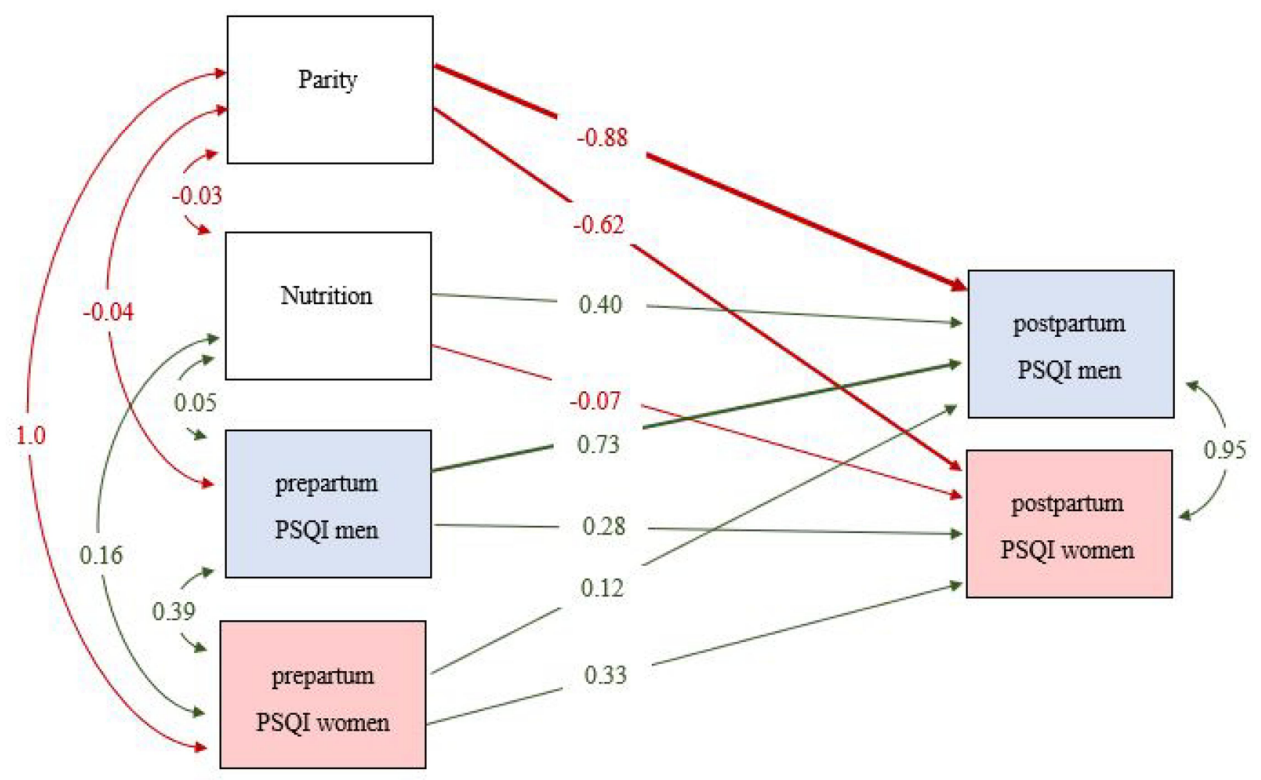

Figure 3 The APIM-covariable model for PSQI with covariates parity and nutrition (Nutrition: reference group= "breast feeding"; Parity: reference group="no children").

sample sizes, measurement time points, and measurement instruments and differed significantly in sample composition.

In the study at hand, men also suffered from sleep deprivation both prenatally and postnatally. Already during pregnancy, expectant fathers reported a lower TST (about $22 \mathrm{~min}$ less than women). These differences persisted after birth (about 21 min less than women). However, these differences may not be related to pregnancy, but rather reflect a gender difference that has been highlighted in previous studies. ${ }^{1,28}$ In summary, in the present study, men were less sleep impaired than women at both measurement time points. This manifested in a lower SOL, a lower WASO a less

Table 3 Couples Distribution in Pregnancy and Postpartum in Sleep Groups in Percent

\begin{tabular}{|l|l|l|}
\hline & Prepartal & Postpartal \\
\hline $\begin{array}{l}\text { Group I } \\
\text { both good sleepers PSQI } \leq 5\end{array}$ & $33.33 \%$ & $39.13 \%$ \\
\hline $\begin{array}{l}\text { Group 2 } \\
\text { one person is a good sleeper and one } \\
\text { person is a bad sleeper PSQI } \leq 5 \text { and } \\
\text { PSQI > 5 }\end{array}$ & $43.48 \%$ & $33.33 \%$ \\
\hline $\begin{array}{l}\text { Group 3 } \\
\text { both bad sleepers PSQI > 5 }\end{array}$ & $23.19 \%$ & $27.54 \%$ \\
\hline
\end{tabular}

FNW, and a higher SE. This result is consistent with other findings that showed quite similar results. ${ }^{4,15,18}$

\section{Mutual Influence in the Sleep of Couples}

Due to the fact that couples influence each other in their sleep behavior, especially when they share a bedroom, it was a particular concern of this study to uncover these influences using an APIM. ${ }^{11}$ The results supported this assumption, but the genders differ significantly. By means of a dyadic data analysis of sleep during pregnancy and the postpartum period, it was possible to prove for the first time that there are differences in the influence of sleep behavior between women and men at pregnancy and postpartum. While in women both their own prenatal PSQI, TST, FNW, and SE co-explain the postpartum PSQI, TST, FNW, and SE, the partners' prenatal PSQI, TST, FNW, and SE also explain the aforementioned postpartum sleep variables in women. Thus, women's postpartum sleep behavior is also influenced by men's prenatal sleep behavior. This underlines the importance of routine examinations during pregnancy to include not only the sleep of the woman but also the sleep of her partner.

In men, however, the picture is different. Their postnatal PSQI, TST, FNW and SE are only influenced by their own prenatal PSQI, TST, FNW and SE and not by the prenatal sleep behavior of the woman. Because no previous studies included dyadic evaluation of couples' sleep in pregnancy and postpartum, the results presented here make a critical 


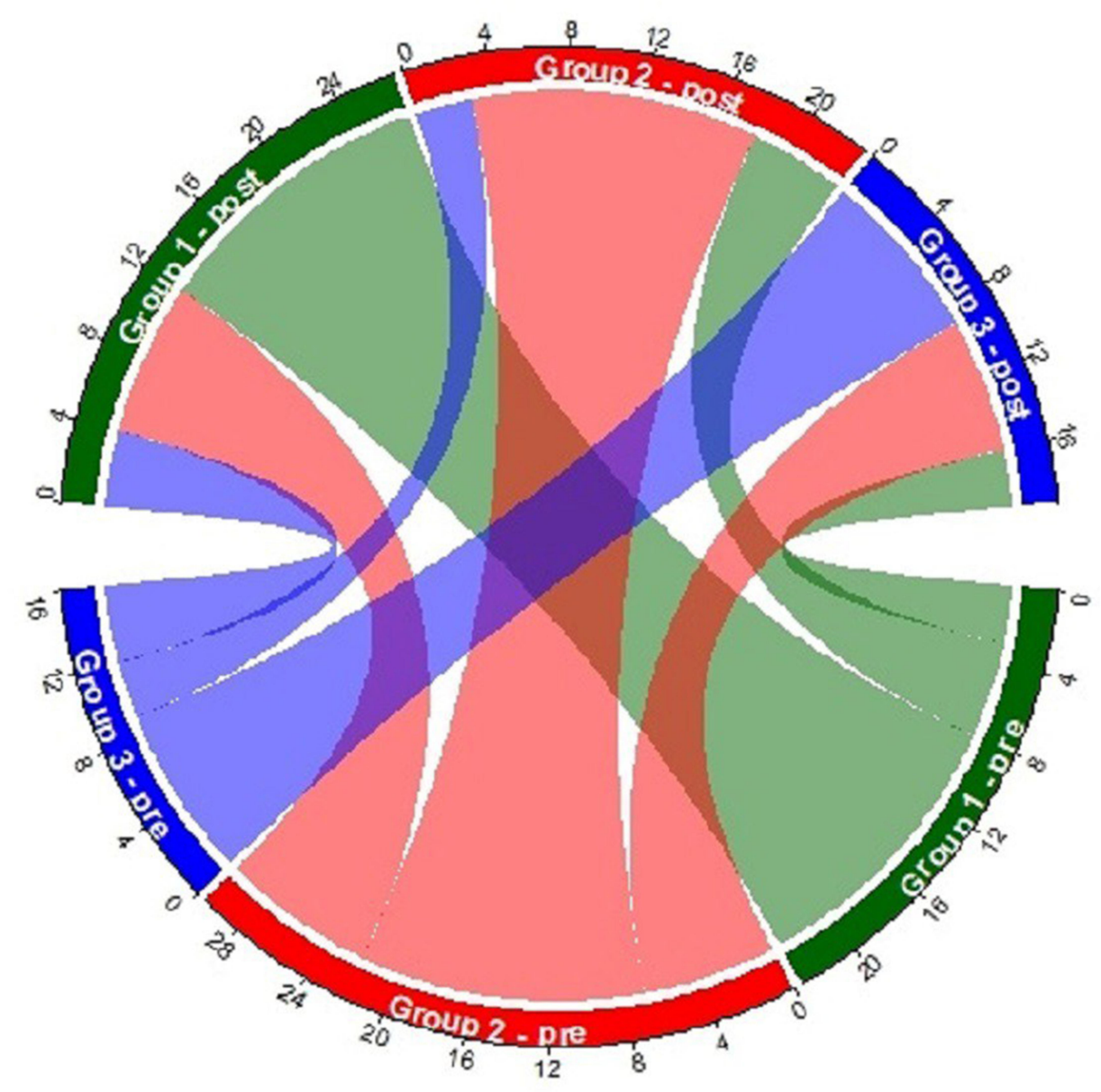

Figure 4 Chord diagram: number of couples separated in sleep groups in pregnancy and the relationship to the postpartal sleep group.

contribution to uncovering sleep problems in couples in transition to parenthood.

\section{Factors Influencing Couples' Sleep}

The hypothesis that multiparous couples sleep better than primiparous couples and parents of breastfed infants sleep better than parents of bottle-fed infants must be rejected. Although multiparous couples had a lower PSQI, as did fathers of breastfed infants, this could not make a decisive contribution to the variance explanation in the postnatal PSQI of mother and father.

\section{The Relationship Between Prenatal and Postnatal Sleep Development of Couples}

Using a chord diagram, it was possible to visualize that most couples in a sleep group in pregnancy remain in that sleep group postnatally, meaning that prenatally poor sleeping couples usually remain postnatally poor sleeping couples. This finding again emphasizes the importance of early identification of sleep problems in couples, so that early intervention can begin during pregnancy to counteract the persistence of sleep difficulties and disorders across the birth.

A strength of this study is its structured approach, which implemented a sleep diary for ten days to provide deep insight into sleep habits and sleep problems in couples both during pregnancy and postpartum. To our knowledge, this is the first study to evaluate couples' sleep in this way. Some researchers have focused only on pregnancy ${ }^{4}$ or the postpartum period, ${ }^{3}$ while others have included pregnancy and the postpartum period. $^{5}$ They all implemented short periods of measurement (2-4 days) in contrast to the current study (10 days). Beside the goal of evaluating the sleep of women and men, the objective was primarily to show that women's and men's sleep cannot be examined independently of one another. 


\section{Limitations}

However, the current study is limited by several factors. One limitation is that all data were based on subjective reports. However, in nonpregnant individuals as well as in pregnancy and postpartum, researchers compared between subjective data, using sleep logs, and objective measures, using actigraphy measurements. ${ }^{13,16,27}$ A study of women over the course of pregnancy and postpartum found minor discrepancies between the two forms of data collection. While TST and SE were slightly overestimated, wake after sleep onset (WASO) was underestimated in the sleep protocols. ${ }^{16}$ In addition, large differences in the measurement of absolute sleep parameters were documented between the two measurement methods, ${ }^{13}$ although the authors attested to large agreements between both methods when used at repeated measurement time points. ${ }^{13}$ Other researchers referred in this context to a possible influence of psychological factors in the subjective recording of sleep quality in the form of sleep protocols in comparison to actigraphy measurement. ${ }^{27}$ They demonstrated a negative effect of perceptions on sleep quality and sleep efficiency with increased daytime sleepiness on the following day when subjective measurement methods were used, not so with actigraphy measurement. ${ }^{27}$

Further, all couples in this study shared a bedroom during pregnancy, and three months postpartum all couples shared a bedroom with their baby. Although this point makes the study more realistic, the results may only be generalizable to couples with the same sleeping habits. Furthermore, the influence of the infant in the shared bedroom with parents should be considered in future studies. Comparisons with other studies are difficult because of varying sample sizes, timeframes, and contents.

\section{Conclusion and Clinical Implications}

This study's findings have significant implications for clinical practice. First of all, all pregnant women and their partner should be routinely assessed for sleep problems. The PSQI could be included into the standard routine of health-care professionals. Hence, existing sleep problems in pregnancy could be detected to prevent severe sleep disorders and chronification. Sleep education programs could be adapted, and effective interventions could be developed to help expectant parents to positively handle their sleeping problems. Early screening and interventions for couples with sleep problems during pregnancy could potentially reduce the risk of chronification of sleep problems. The importance of healthy sleep habits and obtaining sufficient sleep should also be emphasized by prenatal health-care professionals with the aim of improving the postpartal sleep of new parents.

\section{Abbreviations}

FNW, frequency of night waking; SE, sleep efficiency; SOL, sleep onset latency; TST, total sleep time at night; WASO, wake after sleep onset; PSQI, Pittsburgh Sleep Quality Index.

\section{Acknowledgments}

The authors acknowledge support for the publication costs by the Open Access Publication Fund of Bielefeld University. The authors also thank all couples who supported them.

\section{Author Contributions}

All authors made a significant contribution to the work repeated, whether that is in the conception, study design, execution, acquisition of data, analysis and interpretation, or in all these areas; took part in drafting, revising or critically reviewing the article: gave final approval of the version to be published; have agreed on the journal to which the article has been submitted; and agree to be accountable for all aspects of work.

\section{Disclosure}

The authors report no conflicts of interest in this work.

\section{References}

1. Bixler EO, Papaliaga MN, Vgontzas AN, et al. Women sleep objectively better than men and the sleep of young women is more resilient to external stressors: effects of age and menopause. J Sleep Res. 2009;18(2):221-228. doi:10.1111/j.1365-2869.2008.00713.x

2. Buysse DJ, Reynolds CF, Monk TH, Berman SR, Kupfer DJ. The pittsburgh sleep quality index: a new instrument for psychiatric practice and research. Psychiatry Res. 1989;28(2):193-213. doi:10.1016/ 0165-1781(89)90047-4

3. Doan T, Gardiner A, Gay CL, Lee KA. Breast-feeding increases sleep duration of new parents. J Perinat Neonatal Nurs. 2007;21 (3):200-206. doi:10.1097/01.JPN.0000285809.36398.1b

4. Elek SM, Hudson DB, Fleck MO. Expectant parents' experience with fatigue and sleep during pregnancy. Birth. 1997;24(1):49-54. doi:10.1111/j.1523-536X.1997.tb00336.X

5. Gay CL, Lee KA, Lee SY. Sleep patterns and fatigue in new mothers and fathers. Biol Res Nurs. 2004;5(4):311-318. doi:10.1177/ 1099800403262142

6. Gu Z, Gu L, Eils R, Schlesner M, Brors B. Circlize implements and enhances circular visualization in R. Bioinformatics. 2014;30 (19):2811-2812. doi:10.1093/bioinformatics/btu393 
7. Hedman C, Pohjasvaara T, Tolonen U, Suhonen-Malm AS, Myllylä VV. Effects of pregnancy on mothers' sleep. Sleep Med. 2002;3(1):37-42. doi:10.1016/S1389-9457(01)00130-7

8. Hertz G, Fast A, Feinsilver SH, Albertario CL, Schulman H, Fein AM. Sleep in normal late pregnancy. Sleep. 1992;15 (3):246-251. doi:10.1093/sleep/15.3.246

9. Hoffmann DPR, Müller T, Hajak G, Cassel W. Abendmorgenprotokolle in Schlafforschung und Schlafmedizin- ein Standardinstrument für den deutschsprachigen Raum. Somnologie (Berl). 1997;1(3):103-109. doi:10.1007/s11818-997-0019-z

10. Insana SP, Garfield CF, Montgomery-Downs HE. A mixed-method examination of maternal and paternal nocturnal caregiving. J Pediatr Health Care. 2014;28(4):313-321. doi:10.1016/j.pedhc.2013.07.016

11. Kenny DA, Kashy DA, Cook WL. Dyadic Data Analysis. Guilford press; 2006.

12. Lee KA, Zaffke ME, McEnany G. Parity and sleep patterns during and after pregnancy. Obstet Gynecol. 2000;95(1):14-18. doi:10.1016/ S0029-7844(99)00486-X

13. Lockley SW, Skene DJ, Arendt J. Comparison between subjective and actigraphic measurement of sleep and sleep rhythms. J Sleep Res. 1999;8(3):175-183. doi:10.1046/j.1365-2869.1999.00155.x

14. McDaniel BT, Teti DM. Coparenting quality during the first three months after birth: the role of infant sleep quality. J Fam Psychol 2012;26(6):886. doi:10.1037/a0030707

15. Mallampalli MP, Carter CL. Exploring sex and gender differences in sleep health: a society for women's health research report. J Womens Health. 2014;23(7):553-562. doi:10.1089/jwh.2014.4816

16. Matsumoto K, Shinkoda H, Kang MJ, Seo YJ. Longitudinal study of mothers' sleep-wake behaviors and circadian time patterns from late pregnancy to postpartum-monitoring of wrist actigraphy and sleep logs. Biol Rhythm Res. 2003;34(3):265-278. doi:10.1076/brhm.34.3.265.18812

17. Mindell JA, Jacobson BJ. Sleep disturbances during pregnancy. J Obstet Gynecol Neonatal Nurs. 2000;29(6):590-597. doi:10.1111/ j.1552-6909.2000.tb02072.x

18. Mong JA, Cusmano DM. Sex differences in sleep: impact of biological sex and sex steroids. Philos Trans $R$ Soc B. 2016;371 (1688):20150110. doi:10.1098/rstb.2015.0110

19. Perlis ML, Swinkels CM, Gehrman PR, Pigeon WR, MattesonRusby SE, Jungquist CR. The incidence and temporal patterning of insomnia: a pilot study. J Sleep Res. 2010;19(1-Part-I):31-35. doi:10.1111/j.1365-2869.2009.00768.x

20. Pollock MA, Amankwaa LC, Amankwaa AA. First-time fathers and stressors in the postpartum period. J Perinat Educ. 2005;14(2):19-25. doi:10.1624/105812405X44682
21. Quillin SI, Glenn LL. Interaction between feeding method and cosleeping on maternal-newborn sleep. J Obstet Gynecol Neonatal Nurs. 2004;33(5):580-588. doi:10.1177/0884217504269013

22. R Core Team. R: a language and environment for statistical computing. $\mathrm{R}$ foundation for statistical computing. 2020. Vienna, Austria. Available from: https://www.R-project.org/.

23. Richter D, Krämer MD, Tang NK, Montgomery-Downs HE, Lemola S. Long-term effects of pregnancy and childbirth on sleep satisfaction and duration of first-time and experienced mothers and fathers. Sleep. 2019;42(4):zsz015. doi:10.1093/sleep/zsz015

24. Riemann D, Baum E, Cohrs S, Crönlein T, Hajak G, Hertenstein E. $\mathrm{S} 3$ guidelines on non-restorative sleep/sleep disorders. Somnologie. 2017;21(1):2-44. doi:10.1007/s11818-016-0097-x

25. Rogers AE, Caruso CC, Aldrich MS. Reliability of sleep diaries for assessment of sleep/wake patterns. Nurs Res. 1993;42(6):368-371. doi:10.1097/00006199-199311000-00010

26. Rosseel Y. Lavaan: an R package for structural equation modeling and more. Version 0.5-12 (BETA). J Stat Softw. 2012;48(2):1-36. doi:10.18637/jss.v048.i02

27. Russell C, Wearden AJ, Fairclough G, Emsley RA, Kyle SD. Subjective but not actigraphy-defined sleep predicts next-day fatigue in chronic fatigue syndrome: a prospective daily diary study. Sleep. 2016;39(4):937-944. doi:10.5665/sleep.5658

28. Schlarb A, Gulewitsch MD, Weltzer V, Ellert U, Enck P. Sleep duration and sleep problems in a representative sample of German children and adolescents. Health. 2015;07(11):1397-1408. doi:10.4236/health.2015.711154

29. Signal TL, Gander PH, Sangalli MR, Travier N, Firestone RT, Tuohy JF. Sleep duration and quality in healthy nulliparous and multiparous women across pregnancy and post-partum. Aust N Z J Obstet. 2007;47(1):16-22. doi:10.1111/j.1479-828X.2006.00672.x

30. Stas L, Kenny DA, Mayer A, Loeys T. Giving dyadic data analysis away: a user-friendly app for actor-partner interdependence models. Pers Relatsh. 2018;25(1):103-119. doi:10.1111/pere.12230

31. Waters MA, Lee KA. Differences between primigravidae and multigravidae mothers in sleep disturbances, fatigue, and functional status. J Nurs Midwifery. 1996;41(5):364-367. doi:10.1016/S0091-2182(96)00049-3

32. Wulff K, Siegmund R. Circadian and ultradian time patterns in human behaviour: part 1: activity monitoring of families from prepartum to postpartum. Biol Rhythm Res. 2000;31(5):581-602. doi:10.1076/brhm.31.5.581.5658

\section{Publish your work in this journal}

Nature and Science of Sleep is an international, peer-reviewed, open access journal covering all aspects of sleep science and sleep medicine, including the neurophysiology and functions of sleep, the genetics of sleep, sleep and society, biological rhythms, dreaming, sleep disorders and therapy, and strategies to optimize healthy sleep.
The manuscript management system is completely online and includes a very quick and fair peer-review system, which is all easy to use. Visit http://www.dovepress.com/testimonials.php to read real quotes from published authors. 